\title{
Protocatechuic acid attenuates anterior cruciate ligament transection-induced osteoarthritis by suppressing osteoclastogenesis
}

\author{
JIALIN ZHANG $^{1 *}$, BIN FU $^{1 *}$, XIAOLEI CHEN ${ }^{1}$, DESHENG CHEN $^{1}$ and HAO YANG ${ }^{2}$ \\ ${ }^{1}$ Department of Orthopedics, General Hospital of Ningxia Medical University, Ningxia, Gansu 750004; \\ ${ }^{2}$ Department of Orthopedics, The First Affiliated Hospital of Kunming Medical University, \\ Kunming, Yunnan 650032, P.R. China
}

Received June 13, 2018; Accepted October 2, 2019

DOI: $10.3892 /$ etm.2019.8189

\begin{abstract}
Osteoarthritis (OA) is related to over-proliferation or differentiation of osteoclasts. Although protocatechuic acid (PCA) has been identified to inhibit osteoclast differentiation and stimulate apoptosis in mature osteoclasts, whether it can relieve OA is still unknown. The present study aimed to investigate the effect of PCA on anterior cruciate ligament transection (ACLT)-induced $\mathrm{OA}$ and the potential mechanisms of action behind this effect. ACLT was performed on rats, which were then treated with or without PCA. C-terminal telopeptide of type I collagen (CTX-I) and CTX-II were tested in knee joint protein extracts by ELISA. Damage to cartilage was evaluated using Safranin-O/Fast Green staining. Osteoclast-related gene and protein expression was assessed through reverse transcription-quantitative PCR and western blotting. Tartrate-resistant acid phosphatase (TRAP) staining and functional bone resorption pit assays were performed using RAW264.7 murine macrophage cells to determine the effects of PCA on osteoclastic formation and function, respectively, in vitro. Finally, the activity of osteoclastogenesis-related signaling pathways was evaluated by western blotting. Levels of CTX-II were relatively decreased and Safranin-O/fast green staining indicated milder changes in the articular cartilage in the PCA treatment group. PCA downregulated osteoclast specific markers and suppressed receptor activator of nuclear factor- $\kappa \mathrm{B}$ ligand-induced formation of TRAP-positive multinucleated cells, bone-resorption and pit formation. Mitogen-activated protein kinase (MAPK) and Akt signaling as well as the downstream factors, were downregulated by PCA. In conclusion, the present
\end{abstract}

Correspondence to: Dr Hao Yang, Department of Orthopedics, The First Affiliated Hospital of Kunming Medical University, 295 Xichang Road, Kunming, Yunan 650032, P.R. China

E-mail: doctorhaoyang@163.com

*Contributed equally

Key words: protocatechuic acid, osteoarthritis, anterior cruciate ligament transection-induced, osteoclastogenesis study demonstrated that PCA attenuated ACLT-induced OA by suppressing osteoclastogenesis by inhibiting the MAPK, ATK and $\mathrm{NF}-\kappa \mathrm{B}$ signaling pathways.

\section{Introduction}

Osteoarthritis (OA) is a common and costly disease in modern society. It is the leading reason for medical consultation in the elderly and affects $>30 \%$ of people $>60$ years old in the USA $(1,2)$. OA was once considered an articular cartilage disease; however, numerous studies investigating the structures at the osteochondral junction and the pathogenesis/symptoms of OA indicate that it is a 'whole joint disease'. $\mathrm{OA}$ is a pathology with the characteristics of decreased articular cartilage thickness, sclerosis of subchondral bone, formation of osteophytes and alteration of the synovial fluid components $(2,3)$.

Bone tissue is dynamically shaped and repaired. The delicate balance between bone resorption and bone formation is important to maintain normal bone structure and function (4,5). Previous studies have suggested that osteoclast-mediated bone resorption of mineralized cartilage at the interface of subchondral bone and cartilage is an early initiating pathology in the progression of $\mathrm{OA}$, and bisphosphonate can significantly improve the cartilage damage in the early stages (6). Osteoclast-mediated resorption plays a crucial role in $\mathrm{OA}$ and may be an initiator during OA development $(7,8)$. Excessive activation of osteoclasts is considered to be the primary mechanism that leads to joint diseases, including OA, cartilaginous degeneration and osteoporosis $(9,10)$. Thus, inhibition of osteoclastogenesis is likely to be chondroprotective and prevent resorption of cartilage in OA.

Osteoclasts are derived from monocyte/macrophage precursor cells, near to or at the bone surface, and are a tissue-specific macrophage polykaryon (4). Receptor activator of nuclear factor- $\kappa \mathrm{B}$ (RANK) ligand (RANKL) and monocyte/macrophage colony-stimulating factor are haematopoietic factors essential for osteoclastogenesis and bone reabsorption. Once stimulated in the presence of these two cytokines, osteoclast precursors move towards the bone surface and begin to differentiate $(11,12)$. The binding of RANKL with its receptor, 
RANK, induces osteoclast differentiation and activation through numerous signaling cascades, including inhibitor of $\mathrm{NF}-\kappa \mathrm{B}$ kinase (IKK), p38, proto-oncogene tyrosine-protein kinase (c-Src) and JNK pathways. Following osteoclast differentiation, various osteoclastogenesis associated transcription factors, such as NF- $\mathrm{NB}, \mathrm{c}-\mathrm{Fos}$ and nuclear factor of activated T cells 1 (NFATc1), are activated $(13,14)$.

Protocatechuic acid (PCA), also known as 3,4-dihydroxybenzoic acid, is an abundantly and naturally distributed phenolic acid (15). It has been reported to have antioxidant, antibacterial, anti-inflammatory and anti-cancer activity (16-19). Experimental findings demonstrate the promising anti-inflammatory and analgesic activity of PCA in rats and mice, including in a Freund's adjuvant-induced arthritis rat model (19). PCA has recently been found to inhibit the differentiation of osteoclasts and induce apoptosis of mature osteoclasts by regulating oxidative stress and inflammation (20). The present study was designed to investigate the positive effects of PCA on anterior cruciate ligament (ACL) transection (ACLT)-induced OA. Furthermore, an attempt was made to understand the underlying mechanisms of action behind the antiarthritic activity of PCA.

\section{Materials and methods}

Experimental animal model and drug treatment. A total of 72 male Wistar rats (age, 8 weeks; weight, 245-255 g) obtained from Shanghai SLAC Laboratory Animal Co., Ltd. were used in the present study. Rats were housed under controlled conditions $\left(25 \pm 2^{\circ} \mathrm{C} ; 70 \%\right.$ humidity; $12 \mathrm{~h}$ light/dark cycle) with free access to water and food. The experimental protocols were approved by the Animal Experimental Ethical Committee of the General Hospital of Ningxia Medical University and were carried out on the basis of relevant national and international guidelines.

ACLT was generated as previously described (21). Rats were operated on under anesthesia, consisting of an intraperitoneal injection of mixed acepromazine $(1.25 \mathrm{mg} / \mathrm{kg})$, xylazine $(6.25 \mathrm{mg} / \mathrm{kg})$ and ketamine $(38 \mathrm{mg} / \mathrm{kg})(22)$. Incisions were made on the medial side of the right knee joint and the medial side of the patellar tendon with a para-patellar skin incision, successively. The patella was dislocated laterally to gain entry to the joint space, then the ACL was transected when the knee was flexed. Complete transection of the ligament was confirmed by a positive anterior drawer test (23). Then, the joint was washed with sterile saline to minimize the accompanied inflammatory response. Finally, the joint capsule was closed using 3-0 absorbable thread and the skin was sutured with 5-0 nylon thread. Rats in the sham group underwent arthrotomy rather than the full ACLT (21).

All rats were randomly allocated to three groups: Group 1, Sham (arthrotomy); group 2, ACLT; and group 3, ACLT with PCA. Rats within the ACLT with PCA group were administered PCA $(50 \mathrm{mg} / \mathrm{kg}$ ) daily by gavage for 4 consecutive weeks from the day of surgery. The dose of PCA used in the experiment was based upon our own pilot data (Zhang et al; unpublished). Euthanasia was performed on all rats at 1,2 or 4 weeks post-surgery (19).

ELISA. Protein was extracted from knee joints according to Nielsen et al (24) at week 0, 1, 2 and 4 after operation.
Knee joints were isolated after euthanasia as described above, then immediately frozen in liquid nitrogen and stored at $-80^{\circ} \mathrm{C}$ for use. The tibia and femur were cut $3 \mathrm{~mm}$ from the joint, producing samples weighing 500-700 $\mathrm{mg}$. These samples were frozen in liquid nitrogen again, and transferred to a Bessman tissue pulverizer (Spectrum Chemical Manufacturing Corp.). The samples were crushed with $3 \mathrm{ml}$ extraction buffer, consisting of $50 \mathrm{mM}$ Tris- $\mathrm{HCl}$ buffer ( $\mathrm{pH} 7.4$ ), 0.1\% Triton X-100, $0.1 \mathrm{M} \mathrm{NaCl}, 10 \mathrm{Mm}$ (GM6001; Enzo Life Sciences, Inc.) and 1 tablet/10 ml buffer of Complete Mini EDTA-free protease inhibitor cocktail (Roche Diagnostics). Tissues were homogenized twice for $30 \mathrm{sec}$ using an OMNI homogenizer (Omni International, Inc.) at speed level 4 , then the samples were centrifuged for $10 \mathrm{~min}$ at $1,700 \mathrm{x} \mathrm{g}$ and $4^{\circ} \mathrm{C}$, supernatants were obtained and centrifuged at $10,000 \mathrm{x} \mathrm{g}$ and $4^{\circ} \mathrm{C}$. Finally, the supernatants were stored at $-20^{\circ} \mathrm{C}$ until use.

Rat-specific commercially available ELISA kits (Elabscience ${ }^{\circledR}$ ) were used to evaluate the levels of C-terminal telopeptide of type I collagen (CTX)-I (cat no. E-EL-R1456) and CTX-II (cat no. E-EL-R2554) in protein extracts collected from the knee, according to the manufacturer's instructions (24).

Specimen preparation and histological analysis. Knee joints were isolated 4 weeks after surgery. The knee samples were fixed in $4 \%$ formaldehyde in PBS for $48 \mathrm{~h}\left(\mathrm{pH} 7.0 ; 4^{\circ} \mathrm{C}\right)$ and decalcified for $24 \mathrm{~h}$ at $37^{\circ} \mathrm{C}$ with $22.5 \%$ formic acid and $340 \mathrm{mM}$ sodium citrate. Specimens were embedded in paraffin after demineralization. Blocks were trimmed and the articular cartilage was exposed. Sections were collected at intervals of 0,100 and $200 \mu \mathrm{m}$. A total of $105 \mu \mathrm{m}$-thick sections were collected at each interval. These sections were treated with Safranin-O/Fast Green staining as previously described (25).

Total RNA preparation and reverse transcription-quantitative $P C R(R T-q P C R)$. TRIzol ${ }^{\circledR}$ reagent (Invitrogen; Thermo Fisher Scientific, Inc.) was used to extract total RNA from knee tissues following the manufacturer's instructions. cDNA was generated by reverse transcription with equal quantities of RNA using the SuperScript Reverse Transcriptase kit (Thermo Fisher Scientific, Inc.). The PCR was performed under the following conditions: $10 \mathrm{~min}$ at $95^{\circ} \mathrm{C}, 55$ cycles of $15 \mathrm{sec}$ at $95^{\circ} \mathrm{C}$ and $1 \mathrm{~min}$ at $60^{\circ} \mathrm{C}$ and a final $2 \mathrm{~min}$ at $50^{\circ} \mathrm{C}$, were performed with an MX3000P (Stratagene; Agilent Technologies, Inc.). The comparative $2^{-\Delta \Delta \mathrm{Cq}}$ method was used to calculate the relative quantification (26). All qPCR primer sequences were as follows: c-Src forward, 5'-GGACAGTGGCGGATTCTA CAT-3' and reverse, 5'-GGGTCTGAGGCTTGGATGTG-3'; $\beta 3$-integrin forward, 5'-TACTCTGCCTCCACCACCAT-3' and reverse, 5'-TTTCCCGTAAGCATCAACAA-3'); MMP-9 forward, 5'-GCAGAGGCATACTTGTACCG-3' and reverse, 5'-TGATGTTATGATGGTCCCACTTG-3'; IL-6 forward, 5'-GGCCCTTGCTTTCTCTTCG-3' and reverse, 5'-ATAATA AAGTTTTGATTATGT-3'. Total cDNA was amplified and analyzed using SYBR Green PCR Master Mix (Thermo Fisher Scientific, Inc.) in a Fast Real-time PCR 7500 System (Applied Biosystems; Thermo Fisher Scientific, Inc.). The original Cq values were adjusted to $\operatorname{GAPDH}(3,10)$. 
Tartrate-resistance acid phosphatase (TRAP) staining. RAW264.7 cells $\left(1 \times 10^{6}\right.$ cells/well) were cultured in $\alpha$-MEM (Sigma-Aldrich; Merck KGaA) at $37^{\circ} \mathrm{C}$ supplemented with 10\% FBS (Dalian Meilun Biology Technology Co., Ltd.) and RANKL (100 ng/ml) to induce differentiation (27). These cells were treated with $8 \mathrm{mM}$ PCA for $2 \mathrm{~h}$ and continuously treated with RANKL (100 ng/ml) for 4 days. The cells were collected and washed with PBS, then fixed in formalin for $10 \mathrm{~min}$ at room temperature and permeabilized in ethanol/acetone (1:1) for $1 \mathrm{~min}$ at room temperature. The number of TRAP-positive multinucleated cells (MNCs) was observed and images captured using a light microscope at x100 magnification (Leica Microsystems GmBH) $(20,28)$.

Functional bone resorption pit assay. The resorption pit assay was conducted following the methods described by Lu et al (28). RAW264.7 cells ( $1 \times 10^{5}$ cells/well) were seeded at $37^{\circ} \mathrm{C}$ on a 24 -well Corning Osteo Assay Surface well plate (Corning, Inc.), and were pretreated with PCA $(8 \mathrm{mM})$ for $2 \mathrm{~h}$ followed by the addition of $100 \mathrm{ng} / \mathrm{ml}$ RANKL. After 5 days of incubation, all remaining cells were lysed by $\mathrm{NaOH}(1 \mathrm{M})$ for $10 \mathrm{~min}$ at $4^{\circ} \mathrm{C}$, then the wells were washed twice with PBS. The numbers of bone lacuna were observed under a phase contrast inverted microscope (BH-2; Olympus Corporation) and analyzed with Metamorph imaging analysis software version 2.5 at $\mathrm{x} 400$ magnification (Molecular Devices, LLC).

Western blotting. RAW264.7 cells were pretreated with PCA $(8 \mathrm{mM})$ for $2 \mathrm{~h}$ at room temperature and then cultured with RANKL $(100 \mathrm{ng} / \mathrm{ml})$ for $1 \mathrm{~h}$ at $37^{\circ} \mathrm{C}$ [for CTX-I, CTX-II, c-Src, interleukin (IL)-6, mitogen-activated protein kinases (MAPKs) and phosphorylated (p)-Akt expression] or for $3 \mathrm{~h}$ at $37^{\circ} \mathrm{C}$ [for p65, cyclooxygenase (COX)-2 and NFATc1 expression]. RIPA lysis buffer (Sangon Biotech Co., Ltd.) was used to isolate the whole cell protein and a Nuclear Extraction kit (cat. no. Ab113474; Abcam) was used to isolate the nuclear extracts. The bicinchoninic acid method was used to determine protein concentration. Proteins $(100 \mu \mathrm{g})$ were separated by $10 \%$ SDS-PAGE and transferred to PVDF membranes. The membranes were blocked with $5 \%$ powdered non-fat milk for $1 \mathrm{~h}$ at room temperature and were then incubated with antibodies against CTX-I (1:500; cat. no. CEA665Ra; Wuhan Uscn Business Co.,Ltd.),LCTX-II(1:500; cat.no.CEA686Ra;Wuhan Uscn Business Co., Ltd.), MAPK (1:250; cat. no. ab151279; Abcam), Akt (1:250; cat. no. ab151279; Abcam), p-Akt (1:500; cat. no. ab38449; Abcam), COX-2 (1:1,000; cat. no. ab179800; Abcam), NFATc1 (1:1,000; cat. no. ab207215; Abcam), Lamin A/C (1:1,000; cat. no. ab108922, Abcam), $\beta$-actin $(1: 1,000$; cat. no. ab8226; Abcam), c-Src (1:500; cat. no. ab207462; Abcam), IL-6 (1:1,000; cat. no. ab208113; Abcam), NF-кB p65 (1:1,000; cat. no. ab207297; Abcam) overnight at $4^{\circ} \mathrm{C}$. The membranes were further incubated with a 1:10,000 of goat anti-rabbit $\operatorname{IgG}(\mathrm{H}+\mathrm{L})$ horseradish peroxidase conjugate (cat. no. A2028; Beyotime Institute of Biotechnology) as a secondary antibody for $2 \mathrm{~h}$ at room temperature. The immunoreactivity was visualized using an ECL system (Amersham; GE Healthcare). The relative intensity of the signal produced by the western blotting bands was analyzed using ImageJ software (version 1.4.0.; National Institutes of Health) (20).
Statistical analysis. Results in the present study are presented as the mean \pm SEM of three independent experiments. Data comparisons between groups and within groups were analyzed using either unpaired or paired Student's t-test and one-way ANOVA with Tukey's post-hoc test. $\mathrm{P}<0.05$ was considered to indicate a statistically significant difference.

\section{Results}

PCA relieves ACLT-induced OA. To confirm the effect of PCA on collagen degradation and synthesis, two osteoarthritic markers, CTX-I and CTX-II, were detected within whole knee joints from the sham, ACLT and ACLT with PCA-treated groups at weeks $0,1,2$ and 4 after the operation. The levels of CTX-II in the ACLT group were significantly raised 1-4 weeks after surgery compared to the sham control, while this effect was significantly reversed in the ACLT-PCA group (Fig. 1A). This suggested that the rat model of ACLT was successfully established and that PCA had an anti-osteoarthritic effect (Fig. 1A). By contrast, concentrations of CTX-I were not altered during the period after surgery in any group (Fig. 1B). The Safranin-O/Fast Green staining demonstrated decreased proteoglycans in the superficial and even the middle zone, with clefts extending into the middle layers after ACLT surgery. However, the severity of degenerative features in the PCA treatment group was much milder than that in ACLT group, demonstrating that PCA relieved the ACLT-induced degenerative changes in the articular cartilage (Fig. 1C). Collectively, these results indicated that PCA has potential for treating ACLT-induced OA.

PCA decreases the activation of osteoclasts in ACLT rats. To further identify the therapeutic effects of PCA for treating ACLT-induced OA, the mRNA expression of osteoclast-related genes in knee tissues were analyzed by RT-qPCR. Compared with the sham group, the expression levels of c-Src, $\beta 3$-integrin, matrix metalloproteinase (MMP)-9 and IL-6 were significantly increased at the mRNA level after ACLT surgery (Fig. 2A-D). However, PCA treatment effectively reserved these abnormalities. Furthermore, it was also found that the protein levels of CTX-I, CTX-II, c-Src and IL-6 were raised after ACLT surgery compared to the sham-operated group (Fig. 2E). PCA treatment significantly reversed the increased levels of CTX-I, CTX-II, c-Src and IL-6 compared to the ACLT group (Fig. 2E). These results demonstrated that PCA can decrease ACLT-induced osteoclastogenesis in vivo.

PCA suppresses RANKL-induced osteoclast differentiation and the bone resorbing activity of RAW264.7 cells. To observe the effect of PCA on osteoclast differentiation, murine macrophage RAW264.7 cells were incubated in the presence of RANKL with or without PCA. The effect of PCA on osteoclast differentiation was evaluated using TRAP staining. Microscopic assessment showed that abundant TRAP + MNCs were formed in the culture as a response to RANKL on day 4 (Fig. 3A), whereas the PCA treatment reduced the number of RANKL-stimulated TRAP + MNCs cells $(\mathrm{P}<0.01)$. Consistent with the function of preventing osteoclastic differentiation, PCA disrupted the pit-forming activity of RANKL-stimulated osteoclasts. Compared to the group treated with RANKL alone, 
A

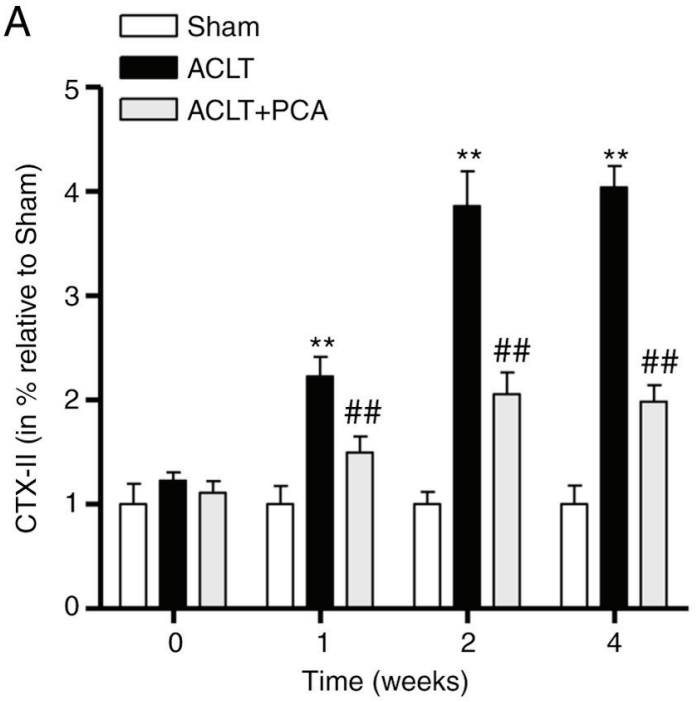

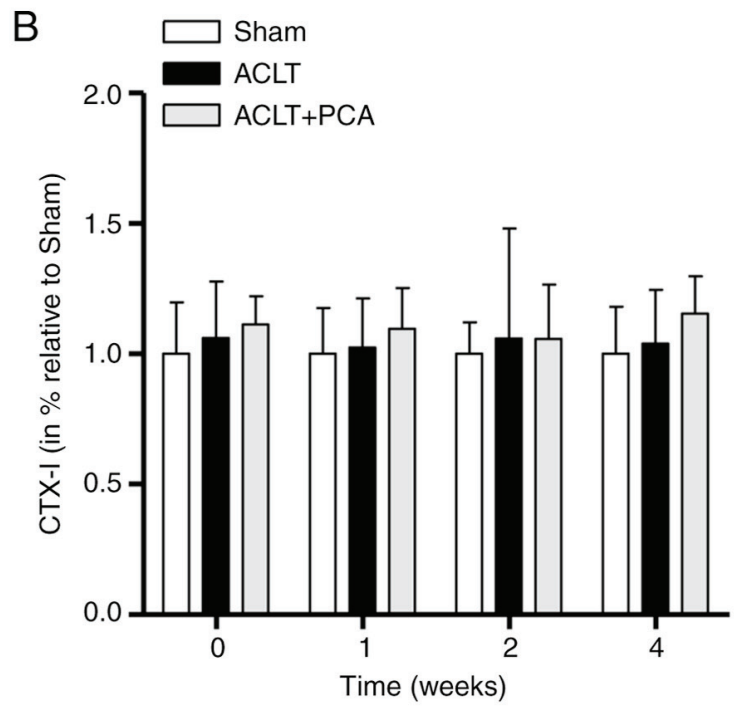

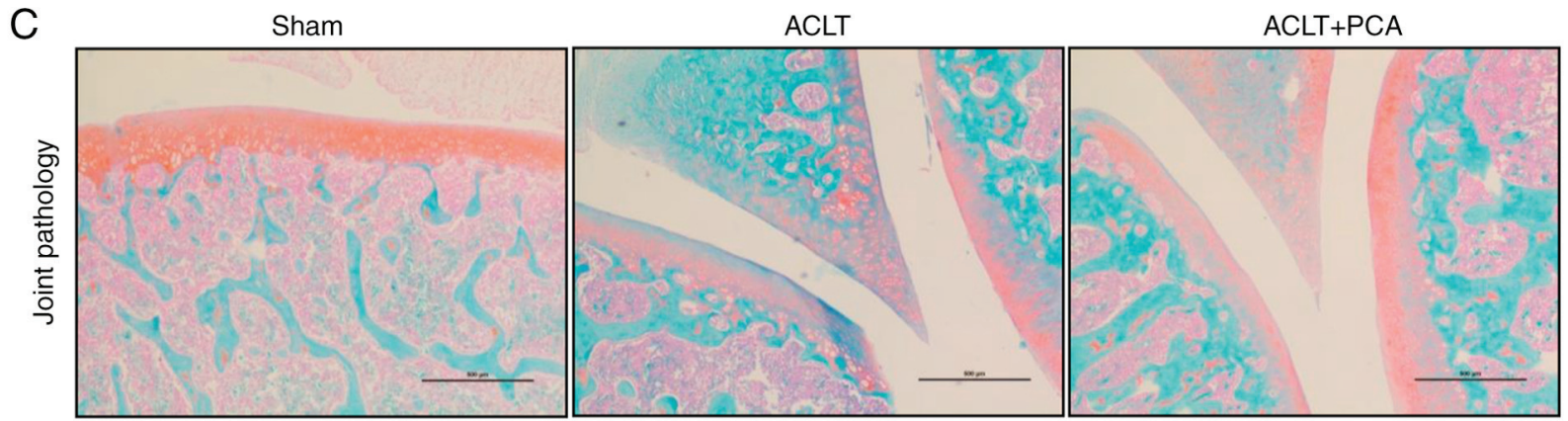

Figure 1. PCA relieves ACLT-induced osteoarthritis. Rats with surgically induced ACLT were treated with or without PCA for 0, 1, 2 and 4 weeks. Levels of (A) CTX-II and (B) CTX-I in the knee joints were tested by ELISA. (C) The degenerative changes in the articular cartilage were examined by Safranin-O/Fast Green staining. Rats undergoing sham surgery were used as a control. The results are shown as the percentage mean \pm SEM (n=6). Statistical analysis: ANOVA with Tukey's post-hoc test; NS, ${ }^{* *} \mathrm{P}<0.01$ vs. sham; ${ }^{\#} \mathrm{P}<0.01$ vs. ACTL. Scale bars, $500 \mu \mathrm{m}$. ACLT, anterior cruciate ligament transection; CTX, C-terminal telopeptide of type I collagen; PCA, protocatechuic acid.

a notable reduction in resorption pit area and number of bone lacuna was observed in the RANKL + PCA group (Fig. 3B). Together, these data demonstrated that PCA suppresses osteoclastogenesis and osteoclast function in vitro. Thereafter, the inhibitory effects of PCA on osteoclastogenesis and bone resorption were further validated in vitro.

PCA inhibited the RANKL-induced activation of MAPK, Akt and $N F-\kappa B$ signaling. In osteoclast precursor cells, the binding of RANKL to its precursor RANK triggers downstream signaling pathways, including MAPKs and Akt signaling pathways which are essential for osteoclast differentiation and activation. In order to investigate the signaling pathways that PCA affects, the expression of MAPKs, p-Akt and the three critical nuclear factors involved in osteoclastogenesis (NF- $\mathrm{B}$ p 65 , c-Fos and NFATc1) were evaluated. The results showed that there was not only a significant increase in the proportion of phosphorylated of MAPKs (ERK, p-38 and JNK) and Akt compared to their unphosphorylated versions, but also increased protein expression levels of NF- $\kappa \mathrm{B}$ p65, c-Fos and NFATc1 (Fig. 4). However, pretreatment with PCA in the present of RANKL led to downregulation in the phosphorylation levels of MAPKs and Akt. Similarly, the downstream nuclear factors (NF- $\mathrm{B}$ p65, c-Fos and NFATc1), crucial for osteoclast differentiation and activation were also downregulated (Fig. 4). These results indicated that PCA suppressed the activation of the MAPK, Akt and NF- $\kappa$ B signaling pathways that were induced by RANKL during osteoclastogenesis.

\section{Discussion}

OA is a prevalent form of arthritic disease, which causes pain and dysfunction principally in the knee and hip joints. OA affects $18 \%$ of women and $9.6 \%$ of men over the age of 60 (29). Patients are burdened with the high medical cost of substantial use of medications. At present, the optimal therapy for OA is a combination of non-pharmacological and pharmacological therapies. Pharmacological therapies are essential to relieve pain and recover joint function for most patients (2). Nonsteroidal anti-inflammatory drugs, corticosteroids and hyaluronan are used clinically to improve the symptoms of OA, but they fail to reverse cartilage damage and they also exhibit a wide range of adverse effects, including cardiovascular, renal and gastrointestinal side effects (30). As such, more effective therapies with milder adverse effects need to be developed.

PCA is a phenolic compound on which a variety of research work has been carried out. Recently, a study demonstrated that PCA has promising anti-inflammatory and analgesic activities in Freund's adjuvant-induced arthritis, because of 
A

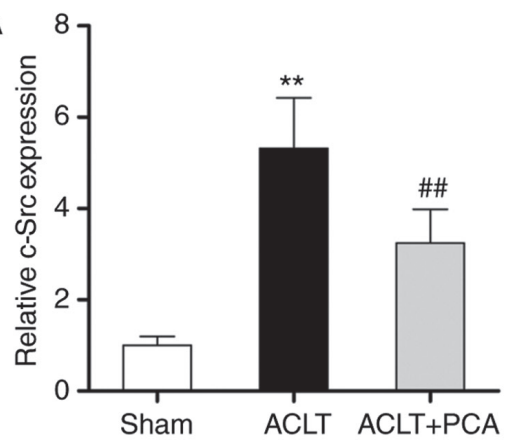

C

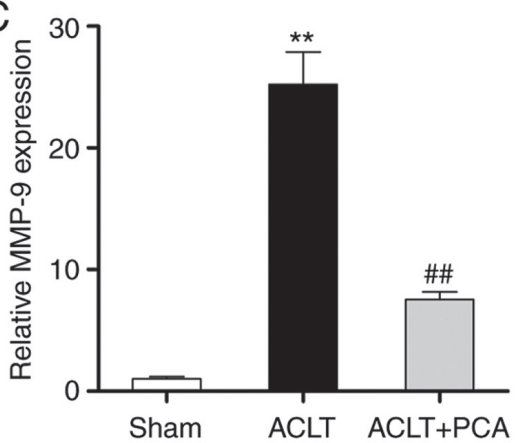

E

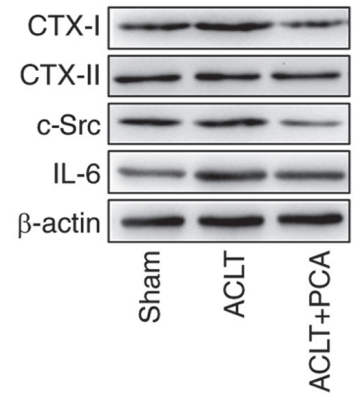

$\mathrm{B}$

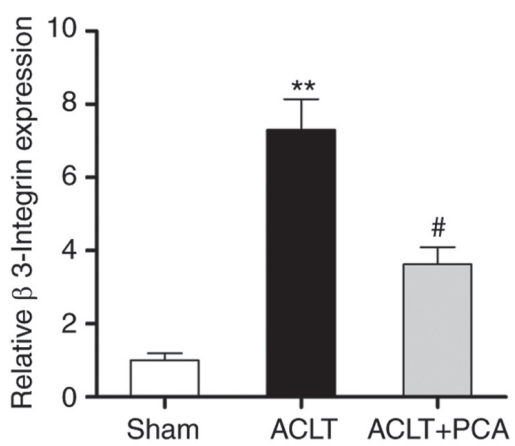

D
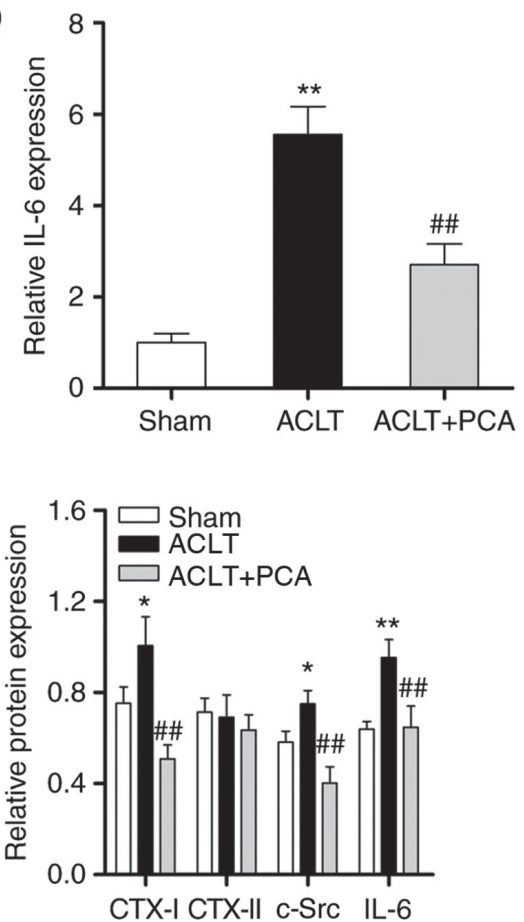

Figure 2. PCA decreases the activation of osteoclast in ACLT rats. The relative expression levels of (A) c-Src, (B) $\beta$-3 Integrin, (C) MMP-9 and (D) IL-6 in knee tissue samples were evaluated through reverse transcription-quantitative PCR. GAPDH was used as a reference. (E) The protein expressions of CTX-I, CTX-II, c-Src and IL-6 in knee tissue samples were evaluated through western blotting. $\beta$-actin was used as a loading control. * $\mathrm{P}<0.05$, ${ }^{* *} \mathrm{P}<0.01$ vs. sham; ${ }^{\#} \mathrm{P}<0.05,{ }^{\#} \mathrm{P}<0.01$ vs. ACTL. ACLT, anterior cruciate ligament transection; c-Src, proto-oncogene tyrosine-protein kinase; MMP, matrix metalloproteinase; PCA, protocatechuic acid.

its antioxidant and membrane-stabilizing properties (19) Another study showed that PCA suppresses osteoclast differentiation induced by RANKL in RAW264.7 cells by regulating oxidative stress and inflammation, and that two osteoclastogenesis-related transcription factors, NF- $\kappa \mathrm{B}$ and Nrf-2, are involved (20). PCA has also been proven to induce apoptosis in mature osteoclasts (20). Importantly, the activation of osteoclasts plays an important role in the initiation and progression of OA. Park et al (31) found that PCA could attenuate osteoclastogenesis by regulating JNK/c-Fos/NFATc1 signaling and preventing inflammatory bone loss in mice. Considering the aforementioned observations, this present study attempted to determine whether PCA can attenuate OA by suppressing osteoclastogenesis mediated by the osteoclastogenesis-associated MAPK and Akt signaling pathways, as well as critical nuclear factors.

In this present study, it was demonstrated that PCA exerts an anti-arthritic effect on ACLT-induced OA rats, a model that has been widely applied to investigate OA. Many biomarkers such as collagens, chondroitin sulfate and aggrecan are used to evaluate OA $(32,33)$. CTX-II is a primary component of articular cartilage and accounts for $90-95 \%$ of collagen content, acting in the formation of the fibrillar structure to maintain the tensile strength of cartilage (33). Thus, CTX-II has been considered as a crucial biomarker for OA in many studies (34). This present research revealed that ALCT surgery substantially raised CTX-II levels in the knee joints compared to the levels in the sham group. Notably, PCA inhibited the degradation of CTX-II induced by ACLT surgery. These results suggested that PCA could regulate the expression of CTX-II during the development of OA. Safranin-O/Fast Green staining demonstrated reduced proteoglycan loss and milder degenerative changes in articular cartilage with PCA treatment. However, consistent with another study (24), the levels of CTX-I were not changed by ACLT or PCA administration. Based on these results, PCA appears to exert a chondroprotective effect on OA induced by 
A

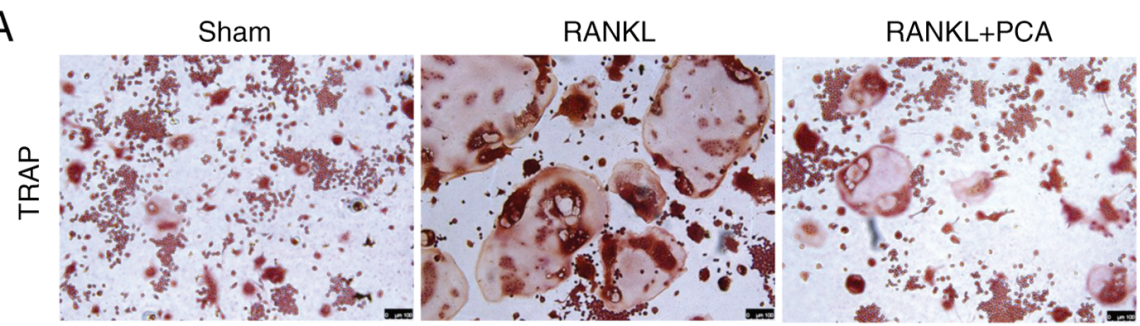

B

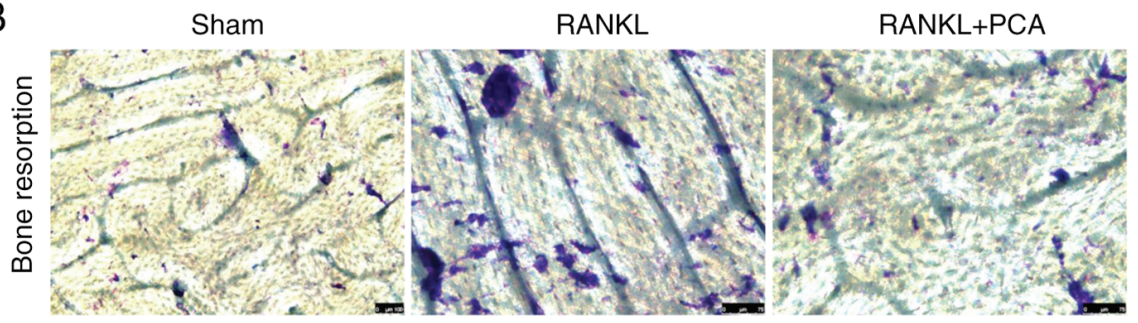

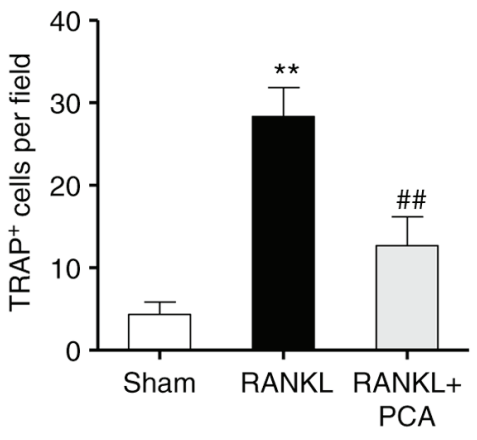

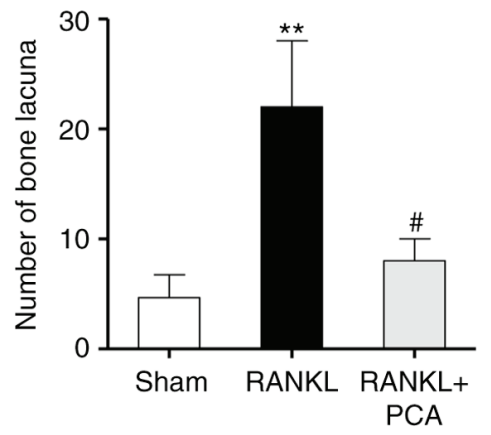

Figure 3. PCA suppresses RANKL-induced osteoclast differentiation and the bone resorbing activity of RAW264.7 cells. (A) The effect of PCA on osteoclast differentiation was evaluated using TRAP staining. The TRAP + multinucleated cells were visualized and counted using an inverted microscope. (B) Pit-forming activity of RANKL-stimulated osteoclasts was analyzed by a microscope. The number of bone lacuna created by osteoclasts was counted. Data are expressed as the mean $\pm \mathrm{SD}(\mathrm{n}=4) .{ }^{* *} \mathrm{P}<0.01 \mathrm{vs}$. sham; ${ }^{\#} \mathrm{P}<0.05,{ }^{\#} \mathrm{P}<0.01 \mathrm{vs}$. RANKL. PCA, protocatechuic acid; RANKL, receptor activator of nuclear factor- $\kappa \mathrm{B}$ ligand; TRAP, tartrate-resistance acid phosphatase.

ACLT by correcting the metabolism of proteoglycans as well as by inhibiting the degradation of CTX-II, and possibly even promoting the synthesis of CTX-II in the cartilage.

Osteoclast activation plays a crucial role in the progression of OA. Inhibiting osteoclast activity prevents bone resorption and can prevent the progression of OA (9). Thus, the expression of osteoclast-related genes in knee tissues was examined in this present study. ACLT enhanced the expression of c-Src, $\beta 3$-integrin and IL- 6 at both the mRNA and protein level. Additionally, Dong et al (35) reported that the absence of MMP-9 restrained the movement of osteoclasts and further affected the functions of osteoclasts. The present study also found that ACLT upregulated the expression of MMP-9. PCA treatment effectively corrected these abnormalities, demonstrating that PCA can reduce osteoclastogenesis in ALCT-induced OA, which may account for the antiarthritic activity of PCA.

To further confirm that PCA suppresses osteoclastogenesis, RAW264.7 osteoclast precursor cells were incubated with PCA and their differentiation induced by RANKL. These data demonstrated that PCA effectively inhibits osteoclast differentiation, reflected by the decrease in TRAP+ MNCs when the cells were treated with both RANKL and PCA, compared to RANKL alone. Osteoclasts are essential for bone resorption which may be enhanced by excessive osteoclastogenesis. A previous study indicated that osteoclast bone resorption is critical in pathological bone diseases including OA (3), and inhibition of osteoclasts has been proven to prevent pain and cartilage degradation in a degenerative joint disease rat model $(7,8)$. In this present study, it was also found that PCA was chondroprotective to the ACLT-induced OA. Thus, it was further speculated that PCA inhibits osteoclast bone resorption. The results from this present study confirmed that PCA significantly decreased the resorption pit area and the number of bone lacuna compared to RANKL treatment alone. Altogether, these in vitro results demonstrated that PCA suppresses osteoclastogenesis and osteoclast function. This conclusion is similar to that of Wu et al (20), who demonstrated that PCA suppresses osteoclast differentiation by negatively regulating osteoclast-related genes and oxidative stress through two crucial transcription factors, NF- $\kappa \mathrm{B}$ and Nrf-2 (20).

RANK/RANKL/osteoprotegerin signaling is an important mediator of osteoclast differentiation and activation $(36,37)$. TNF receptor associated factor 6 (TRAF6) is a key adaptor that assembles signaling proteins and triggers the expression of osteoclast-specific genes, resulting in osteoclast differentiation and activation $(38,39)$. Among those known signaling cascades involved in osteoclast differentiation and activation, MAPK signaling is critical for osteoclast differentiation and has been relatively well characterized (40). c-Src plays an important role in osteoclast activation, by binding to TRAF6 and delivering RANK-induced signaling to PI3K and Akt, promoting cell survival, cytoskeletal rearrangement and cellular motility $(38,39,41)$. Many downstream molecules of $\mathrm{PI} 3 \mathrm{~K} / \mathrm{Akt}$, such as mTOR, protein tyrosine kinase $2 \beta$ and $\mathrm{CBL}$, are known to induce osteoclast differentiation, survival and bone resorption $(40,42,43)$. The $N F-\kappa B$ pathway is also involved in osteoclast activation and the subsequent bone resorption. Upon RANKL stimulation, IKK phosphorylates $\mathrm{p} 65$, which is then translocated to the nucleus (44). NF- $\kappa \mathrm{B}$ regulates the expression of c-Fos (45) and NFATc1 (46) in RANKL-mediated osteoclastogenesis. MAPKs also regulate the RANKL-induced activation of AP-1 through c-Fos and 
A
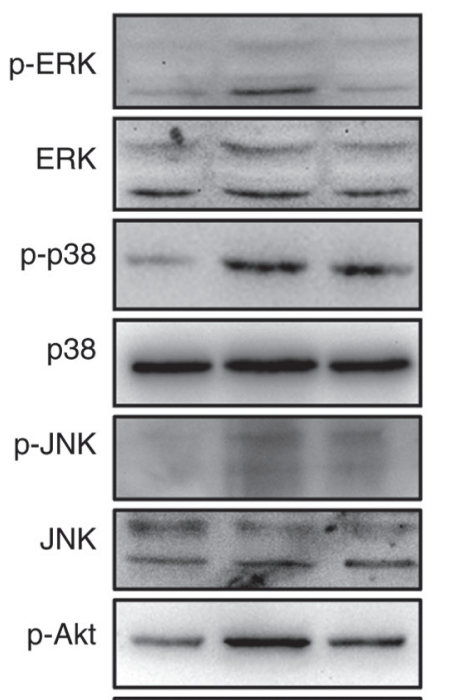

Akt

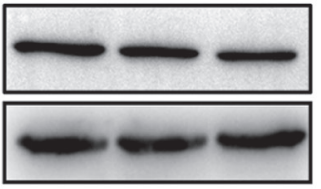

$\beta$-actin

Sham RANKL RANKL+ PCA

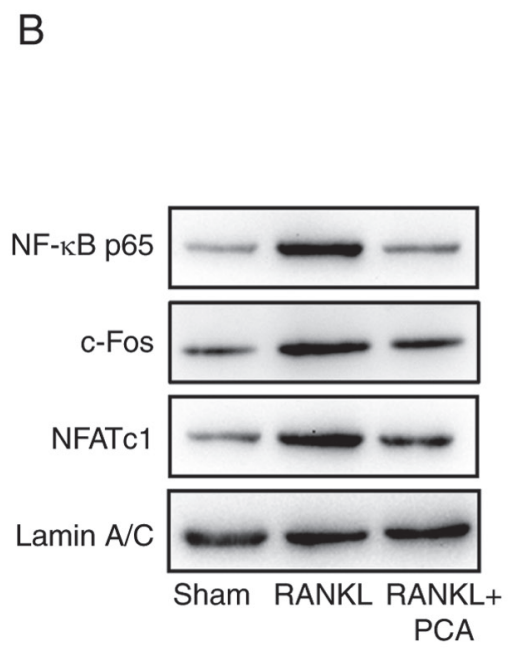

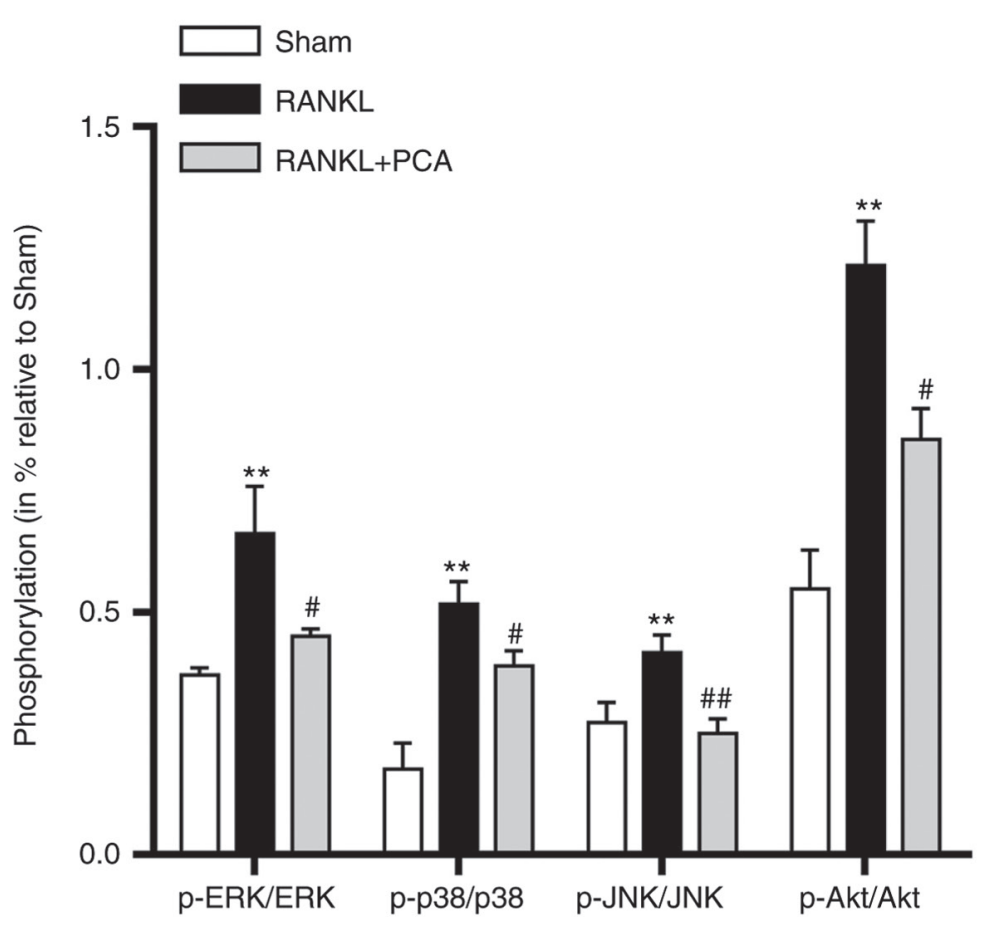

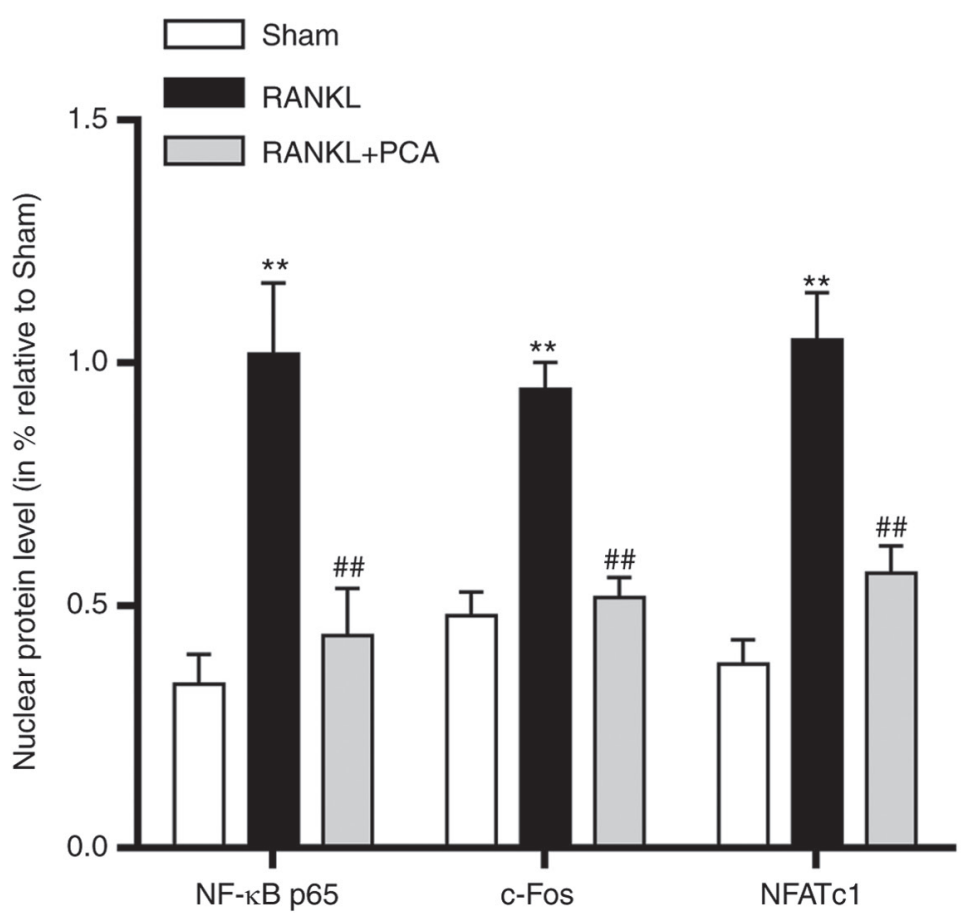

Figure 4. PCA negatively regulates the osteoclastogenesis-associated MAPK and Akt signaling pathways and critical nuclear factors. RAW 264.7 cells were treated with PCA for $2 \mathrm{~h}$ and cultured with RANKL for the corresponding time. (A) Western blotting was used to analyze the total protein extracts of proteins associated with the MAPKs and Akt signaling pathways. Phosphorylation of MAPKs (ERK, p-38 and JNK) and Akt was quantified. (B) Western blotting was used to analyze the expression of p65, c-Fos and NFATc1. All data represent at least three independent experiments. Values represent the mean \pm SEM. ${ }^{* *} \mathrm{P}<0.01$ vs. sham; ${ }^{\#} \mathrm{P}<0.05,{ }^{\# \#} \mathrm{P}<0.01$ vs. RANKL. MAPK, mitogen-activated protein kinases; NFATc1, nuclear factor of activated T cells 1; $\mathrm{p}$, phosphorylated; PCA, protocatechuic acid; RANKL, receptor activator of nuclear factor-kB ligand.

NFATc1. The two factors work synergistically to promote the expression of terminal osteoclastogenesis-related genes (44). Consistent with a previous study (20), the results of this present study demonstrated that all three MAPK families, ERK, JNK and $\mathrm{p} 38$, as well as Akt, were activated by RANKL in osteoclast precursors. The downstream nuclear factors p65, c-Fos and NFATc1, essential for osteoclastogenesis, were also activated.
Nevertheless, PCA effectively inhibited the phosphorylation of MAPKs and Akt induced by RANK signaling. Likewise, the critical downstream factors p65, c-Fos and NFATc1 were suppressed by PCA.

There are several limitations related to the current study. For example, PCA can promote the proliferation and phenotypic maintenance of rabbit articular chondrocytes (47). 
However, the effect of PCA on chondrocytes has not been assessed in the ACLT-induced OA rat model. Additionally, the balance between osteoblasts and osteoclasts is important for the progression of OA. It has been proven that PCA also can promote the proliferation and differentiation of primary rat osteoblasts cultured in vitro (48). This present study aimed to determine the effects of PCA on osteoclasts in OA; the effects of PCA on osteoblasts in OA will be the focus of future studies. It is planned for future research to use MAPK, Akt or NF- $\mathrm{NB}$ agonists for rescue experiments in osteoclasts after PCA treatment. Changes in related phenotypes and biomarkers, such as osteoclast proliferation and differentiation will be examined in further in vitro experiments.

In conclusion, this present study suggested that PCA can efficiently attenuate ALCT-induced OA. PCA not only suppressed the formation of osteoclasts, but also interrupted osteoclast bone resorption. It was found that osteoclastogenesis-associated signaling, including MAPK, Akt and NF- $\mathrm{BB}$ signaling, as well as the critical nuclear factors induced by RANKL, were suppressed by PCA. Thus, the anti-arthritic effect of PCA is primarily associated with the inhibition effect of osteoclastogenesis. Therefore, it was indicated that PCA has the potential to be developed as a new therapy for OA and other diseases associated with excessive osteoclast differentiation and activation.

\section{Acknowledgements}

Not applicable.

\section{Funding}

No funding was received.

\section{Availability of data and materials}

All data generated or analyzed in this study are available from the corresponding author on reasonable request.

\section{Authors' contributions}

HY conceived and designed the study. JZ and BF performed the experiments and collected data. XC and DC were responsible for statistical analysis and literature research. JZ, BF and $\mathrm{XC}$ were major contributors in writing the manuscript and data interpretation. HY had full access to all data in the study and took responsibility for the integrity of the data and the accuracy of the data analysis. All authors read and approved the final manuscript.

\section{Ethics approval and consent to participate}

The experimental protocols were approved by the Animal Experimental Ethical Committee of the General Hospital of Ningxia Medical University and were carried out on the basis of relevant national and international guidelines.

\section{Patient consent for publication}

Not applicable.

\section{Competing interests}

The authors declare that they have no competing interests.

\section{References}

1. Lluch Girbés E, Nijs J, Torres-Cueco R and López Cubas C: Pain treatment for patients with osteoarthritis and central sensitization. Phys Ther 93: 842-851, 2013.

2. Clouet J, Vinatier C, Merceron C, Pot-vaucel M, Maugars Y, Weiss P, Grimandi G and Guicheux J: From osteoarthritis treatments to future regenerative therapies for cartilage. Drug Discov Today 14: 913-925, 2009.

3. Suri S and Walsh DA: Osteochondral alterations in osteoarthritis. Bone 51: 204-211, 2012.

4. Boyle WJ, Simonet WS and Lacey DL: Osteoclast differentiation and activation. Nature 423: 337-342, 2003.

5. Rodan GA and Martin TJ: Therapeutic approaches to bone diseases. Science 289: 1508-1514, 2000.

6. Koh YH, Hong SH, Kang HS, Chung CY, Koo KH, Chung HW, Cha JH and Son KR: The effects of bone turnover rate on subchondral trabecular bone structure and cartilage damage in the osteoarthritis rat model. Rheumatol Int 30: 1165-1171, 2010.

7. Strassle BW, Mark L, Leventhal L, Piesla MJ, Jian Li X, Kennedy JD, Glasson SS and Whiteside GT: Inhibition of osteoclasts prevents cartilage loss and pain in a rat model of degenerative joint disease. Osteoarthritis Cartilage 18: 1319-1328, 2010.

8. Bagi CM, Berryman E, Zakur DE, Wilkie D and Andresen CJ: Effect of antiresorptive and anabolic bone therapy on development of osteoarthritis in a posttraumatic rat model of OA. Arthritis Res Ther 17: 315, 2015.

9. Siebelt M, Waarsing JH, Groen HC, Müller C, Koelewijn SJ, de Blois E, Verhaar JA, de Jong $M$ and Weinans H: Inhibited osteoclastic bone resorption through alendronate treatment in rats reduces severe osteoarthritis progression. Bone 66: 163-170, 2014.

10. Zhao C, Liu Q and Wang K: Artesunate attenuates ACLT-induced osteoarthritis by suppressing osteoclastogenesis and aberrant angiogenesis. Biomed Pharmacother 96: 410-416, 2017.

11. Yasuda H, Shima N, Nakagawa N, Yamaguchi K, Kinosaki M, Mochizuki S, Tomoyasu A, Yano K, Goto M, Murakami A, et al: Osteoclast differentiation factor is a ligand for osteoprotegerin/osteoclastogenesis-inhibitory factor and is identical to TRANCE/RANKL. Proc Natl Acad Sci USA 95: 3597-3602, 1998.

12. Lacey DL, Timms E, Tan HL, Kelley MJ, Dunstan CR, Burgess T, Elliott R, Colombero A, Elliott G, Scully S, et al: Osteoprotegerin ligand is a cytokine that regulates osteoclast differentiation and activation. Cell 93: 165-176, 1998.

13. Zhao Q, Wang X, Liu Y, He A and Jia R: NFATc1: Functions in osteoclasts. Int J Biochem Cell Biol 42: 576-579, 2010

14. Takayanagi H, Kim S, Koga T, Nishina H, Isshiki M, Yoshida H, Saiura A, Isobe M, Yokochi T, Inoue J, et al: Induction and activation of the transcription factor NFATc1 (NFAT2) integrate RANKL signaling in terminal differentiation of osteoclasts. Dev Cell 3: 889-901, 2002.

15. Kakkar S and Bais S: A review on protocatechuic acid and its pharmacological potential. ISRN Pharmacol 2014: 952943, 2014.

16. Li XC, Wang XZ, Chen DF and Chen SZ: Antioxidant activity and mechanism of protocatechuic acid in vitro. Funct Foods Health Dis 1: 232-244, 2011.

17. Mahadevan N, Shivali and Kamboj P: Hibiscus sabdariffa Linn.-An overview. Nat Prod Radiance 8: 77-83, 2009.

18. Tanaka T, Tanaka T and Tanaka M: Potential cancer chemopreventive activity of protocatechuic acid. J Exp Clin Med 3: 27-33, 2011.

19. Lende AB, Kshirsagar AD, Deshpande AD, Muley MM, Patil RR, Bafna PA and Naik SR: Anti-inflammatory and analgesic activity of protocatechuic acid in rats and mice. Inflammopharmacology 19: 255-263, 2011.

20. Wu YX, Wu TY, Xu BB, Xu XY, Chen HG, Li XY and Wang G: Protocatechuic acid inhibits osteoclast differentiation and stimulates apoptosis in mature osteoclasts. Biomed Pharmacother 82: 399-405, 2016.

21. Galois L, Etienne S, Grossin L, Watrin-Pinzano A, CournilHenrionnet C, Loeuille D, Netter P, Mainard D and Gillet P: Dose-response relationship for exercise on severity of experimental osteoarthritis in rats: A pilot study. Osteoarthritis Cartilage 12: 779-786, 2004. 
22. Welberg LA, Kinkead B, Thrivikraman K, Huerkamp MJ, Nemeroff CB and Plotsky PM: Ketamine-xylazine-acepromazine anesthesia and postoperative recovery in rats. J Am Assoc Lab Anim Sci 45: 13-20, 2006.

23. Nyska M, Amir H, Porath A and Dekel S: Radiological assessment of a modified anterior drawer test of the ankle. Foot Ankle 13: 400-403, 1992.

24. Nielsen RH, Stoop R, Leeming DJ, Stolina M, Qvist P, Christiansen C and Karsdal MA: Evaluation of cartilage damage by measuring collagen degradation products in joint extracts in a traumatic model of osteoarthritis. Biomarkers 13: 79-87, 2008.

25. Zhang Z, Wei X, Gao J, Zhao Y, Zhao Y, Guo L, Chen C, Duan Z, Li P and Wei L: Intra-Articular injection of cross-linked hyaluronic acid-dexamethasone hydrogel attenuates osteoarthritis: An experimental study in a rat model of osteoarthritis. Int J Mol Sci 17: 411, 2016.

26. Livak KJ and Schmittgen TD: Analysis of relative gene expression data using real-time quantitative PCR and the 2(-Delta Delta C(T)) method. Methods 25: 402-408, 2001

27. Mau LP, Cheng WC, Chen JK, Shieh YS, Cochran DL and Huang RY: Curcumin ameliorates alveolar bone destruction of experimental periodontitis by modulating osteoclast differentiation, activation and function. J Funct Foods 22: 243-256, 2016

28. Lu SH, Huang RY and Chou TC: Magnolol ameliorates ligature-induced periodontitis in rats and osteoclastogenesis: In vivo and in vitro study. Evid Based Complement Alternat Med 2013: $634095,2013$.

29. Woolf AD and Pfleger B: Burden of major musculoskeletal conditions. Bull World Health Organ 81: 646-656, 2003.

30. Farkouh ME, Greenberg JD, Jeger RV, Ramanathan K, Verheugt FW, Chesebro JH, Kirshner H, Hochman JS, Lay CL, Ruland S, et al: Cardiovascular outcomes in high risk patients with osteoarthritis treated with ibuprofen, naproxen or lumiracoxib. Ann Rheum Dis 66: 764-770, 2007.

31. Park SH, Kim JY, Cheon YH, Baek JM, Ahn SJ, Yoon KH, Lee MS and Oh J: Protocatechuic acid attenuates osteoclastogenesis by downregulating JNK/c-Fos/NFATc1 signaling and prevents inflammatory bone loss in mice. Phytother Res 30 : 604-612, 2016.

32. Rousseau JC and Delmas PD: Biological markers in osteoarthritis. Nat Clin Pract Rheumatol 3: 346-356, 2007.

33. Garnero P, Rousseau JC and Delmas PD: Molecular basis and clinical use of biochemical markers of bone, cartilage, and synovium in joint diseases. Arthritis Rheum 43: 953-968, 2000.

34. Garnero P, Piperno M, Gineyts E, Christgau S, Delmas PD and Vignon E: Cross sectional evaluation of biochemical markers of bone, cartilage, and synovial tissue metabolism in patients with knee osteoarthritis: Relations with disease activity and joint damage. Ann Rheum Dis 60: 619-626, 2001.

35. Dong Z, Bonfil RD, Chinni S, Trindade Filho JC, Bhagat S, Bhagat S, Fridman R and Cher ML: Enhanced osteoclast motility due to overexpression of MMP-9 induced by prostate cancer cells. Cancer Res 64: 773-773, 2004.

36. Anderson DM, Maraskovsky E, Billingsley WL, Dougall WC Tometsko ME, Roux ER, Teepe MC, DuBose RF, Cosman D and Galibert L: A homologue of the TNF receptor and its ligand enhance T-cell growth and dendritic-cell function. Nature 390: 175-179, 1997.
37. Hsu H, Lacey DL, Dunstan CR, Solovyev I, Colombero A, Timms E, Tan HL, Elliott G, Kelley MJ, Sarosi I, et al: Tumor necrosis factor receptor family member RANK mediates osteoclast differentiation and activation induced by osteoprotegerin ligand. Proc Natl Acad Sci USA 96: 3540-3545, 1999.

38. Franzoso G, Carlson L, Xing L, Poljak L, Shores EW, Brown KD, Leonardi A, Tran T, Boyce BF and Siebenlist U: Requirement for NF-kappaB in osteoclast and B-cell development. Genes Dev 11: 3482-3496, 1997.

39. Grigoriadis AE, Wang ZQ, Cecchini MG, Hofstetter W, Felix R, Fleisch HA and Wagner EF: C-Fos: A key regulator of osteoclast-macrophage lineage determination and bone remodeling. Science 266: 443-448, 1994.

40. Lee ZH and Kim HH: Signal transduction by receptor activator of nuclear factor kappa B in osteoclasts. Biochem Biophys Res Commun 305: 211-214, 2003.

41. Wong BR, Besser D, Kim N, Arron JR, Vologodskaia M, Hanafusa $\mathrm{H}$ and Choi Y: TRANCE, a TNF family member, activates Akt/PKB through a signaling complex involving TRAF6 and c-Src. Mol Cell 4: 1041-1049, 1999.

42. Kolbe L, Immeyer J, Batzer J, Wensorra U, tom Dieck K, Mundt C, Wolber R, Stäb F, Schönrock U, Ceilley RI and Wenck H: Anti-inflammatory efficacy of Licochalcone A: Correlation of clinical potency and in vitro effects. Arch Dermatol Res 298: 23-30, 2006.

43. Shui C,Riggs BLand Khosla S: Theimmunosuppressant rapamycin, alone or with transforming growth factor-beta, enhances osteoclast differentiation of RAW264.7 monocyte-macrophage cells in the presence of RANK-ligand. Calcif Tissue Int 71: 437-446, 2002.

44. Kim WS, Kim HJ, Lee ZH, Lee Y and Kim HH: Apolipoprotein $\mathrm{E}$ inhibits osteoclast differentiation via regulation of c-Fos, NFATc1 and NF-кB. Exp Cell Res 319: 436-446, 2013.

45. Yamashita T, Yao Z, Li F, Zhang Q, Badell IR, Schwarz EM, Takeshita S, Wagner EF, Noda M, Matsuo K, et al: NF-kappaB p50 and p52 regulate receptor activator of NF-kappaB ligand (RANKL) and tumor necrosis factor-induced osteoclast precursor differentiation by activating c-Fos and NFATc1. J Biol Chem 282: 18245-18253, 2007.

46. Takatsuna H, Asagiri M, Kubota T, Oka K, Osada T, Sugiyama C, Saito H, Aoki K, Ohya K, Takayanagi $\mathrm{H}$ and Umezawa $\mathrm{K}$ : Inhibition of RANKL-induced osteoclastogenesis by (-)-DHMEQ, a novel NF-kappaB inhibitor, through downregulation of NFATc1. J Bone Miner Res 20: 653-662, 2005.

47. Luo L, Wei Q, Liu L, Lin X, Lin C, Zheng LI and Zhao J: Protocatechuic acid benefits proliferation and phenotypic maintenance of rabbit articular chondrocytes: An study. Exp Ther Med 9: 1865-1870, 2015.

48. Chin A, Yang Y, Chai L, Wong RW and Rabie AB: Effects of medicinal herb salvia miltiorrhiza on osteoblastic cells in vitro. J Orthop Res 29: 1059-1063, 2011

(i) $\Theta$ This work is licensed under a Creative Commons Attribution-NonCommercial-NoDerivatives 4.0 International (CC BY-NC-ND 4.0) License. 